\title{
Applying Ramseyfication to Infrared Spectroscopy
}

\author{
Michael Toppel ${ }^{1}$ (C)
}

Received: 4 November 2020 / Accepted: 7 December 2021

(c) The Author(s) 2021

\begin{abstract}
The so-called Ramsey-Carnap approach, or Ramseyfication, has gone out of fashion in the philosophy of science. Advocates have tried to argue for a revival by writing methodological and metatheoretical studies of Ramseyfication. For this paper I have chosen a different approach; I will apply Ramseyfication to infrared spectroscopya method used in analytical chemistry-in order to logically analyse the relation between measurements and mathematical structures. My aim in doing so is to contribute to the debate about the Application Problem of Mathematics, thereby making a case for Ramseyfication as a method of study for scientific theories.
\end{abstract}

\section{Introduction}

The aim of the present text is to show that the Ramsey-Carnap approach (or Ramseyfication) can be successfully applied to problems in contemporary philosophy, specifically the Application Problem of Mathematics as introduced by Wigner. ${ }^{1}$ [See Wigner (1960).] My aim is not to introduce the Application Problem in full detail or to develop solutions to every aspect of it. The focus here is on adapting and developing the Ramsey-Carnap method further to provide interesting partial solutions to this particular problem. Neither am I particularly keen on conducting a metatheoretical analysis of the Ramsey-Carnap method. Partly because this has already been done in numerous publications by Lutz (2014b, 2017), and partly because I believe that methods such as logical analysis or rational reconstruction reveal their benefits when they are applied to interesting examples. The example in this case is infrared spectroscopy, a common technique for qualitative analysis in organic chemistry that provides directs links between theoretical chemistry-i.e. applied quantum mechanics - and the practices of scientists working in a laboratory. This link makes infrared spectroscopy an ideal element of scientific practice for finding interesting

\footnotetext{
1 The content of this paper was extracted from my thesis. (Toppel 2020)
}

Michael Toppel

michael.toppel@univie.ac.at

1 Institute of Philosophy, University of Vienna, Vienna, Austria 
correspondence rules, as Carnap called the logical bridges between theoretical and observational (or empirical) content of scientific theories.

Historically, Rudolf Carnap's aim in separating theoretical and observational content was to carve out the structural properties that were hidden in the theoretical content and required to make a theory empirically adequate. ${ }^{2}$ The guiding idea of Ramseyfication, i.e. dividing the language of a theory into an observational and a theoretical part, provided the means to perform such a separation. Carnap's original intention was to eventually eliminate the ontological commitments to the referents of the theoretical part by characterising these entirely through structural properties. [See Psillos (1999), p. 47-48 and Uebel (2011) for more on these topics.]

Some philosophers have tried to interpret this as a way of eliminating ontological commitments to any unobservable entity, including abstract objects. These philosophers therefore regard mathematical terms as belonging to the theoretical vocabulary of a language. [See Psillos (1999, p. 47).] Note that, instead, I will be following Carnap's original view by including terms of elementary mathematics in the observational language as presented in Sect. 4. This reconstitution of Carnap's original idea will prove to be essential when it comes to understanding how results from pure mathematics apply to quantified data and measurements.

To align mathematics with the structural nature of the Ramsey-Carnap method, I adopt the stance on mathematics proposed by Maddy (2008). In Maddy's view, mathematics is a kind of warehouse of structures that can be applied to the sciences and (reused) in mathematics itself. She argues in favour of this view historically, dividing the history of mathematics into three phases: antiquity, the period after the scientific revolution up to the development of modern algebra, and the phase of modern algebra. The connection between mathematics and the sciences was severed during the transition from the second to the third phase, as mathematicians became more occupied with studying structures for reasons specific to their discipline. Hence, mathematics can offer these structures for other disciplines to use as they please. As Maddy also states in (2008, p. 35), she views this as support for her criticism on the indispensability argument for mathematical realism as proposed by W.V.O. Quine and H. Putnam, which is one of her central tenets in Second Philosophy. [See Maddy (2007, IV.2).] Both authors argue in favour of a kind of holism in which mathematics and the sciences form a contiguous whole, because the references to mathematical entities cannot be eliminated from their contexts. ${ }^{3}$ I agree with Maddy's historical description of mathematics. Moreover, my use of Ramseyfication will support her critique of the indispensability argument for mathematical realism. Even if the reader disagrees, Maddy's view is surely influential and fits

\footnotetext{
${ }^{2}$ I will not be clarifying the meaning of "structural content" in this paper because there is no need to choose an account of structuralism at this point. In fact, my stance might even provide the grounds to attack structuralism in a novel way.

${ }^{3}$ Note that Patrick Suppes also holds that mathematics cannot be practically separated from the theoretical sciences in Suppes (2002, p.33).
} 
perfectly into the Ramsey-Carnap tradition, which shows that the received view can still contribute to current debates. ${ }^{4}$

There is another peculiarity to my approach besides my opposition to mathematical terms being excluded from the observational part of a theory. Given that my aim is to enlighten on the Application Problem of Mathematics, I cannot conflate formalisation with the application of mathematics from the beginning. ${ }^{5}$ Therefore, I will desist from targeting specific mathematical entities as referents when formalising, i.e. when formulating a syntax and axioms. But since physical phenomena (e.g. temperatures) and numbers (e.g. 20 degrees centigrade) are frequently linked, I must handle identifications like these in a different way. Rather than viewing these links as mere identities, I focus on the measurement unit used to link these elements. In Sect. 5, therefore, I will introduce a logic of measurement units. I do not want to raise any false hopes though; there will neither be a formalisation nor a discussion of the logical properties of measurement units. However, since the lack of a clear picture of the logical properties of units is ultimately unproblematic, their logical behaviour can be ignored. In fact, one major finding of this paper is that the application of mathematics justifies this ignorance. And I would claim that this is an essential element of the application of mathematics to the sciences.

Moreover, I would argue that my framework incorporates the problem of units adequately, because the possibility for units to be ignored here at least partly explains why the logic of units does not appear as a problem in the praxis of the sciences.

The paper will proceed as follows: Sect. 2 will introduce the Application Problem of Mathematics, then in Sect. 3 I will provide a technical introduction to the Ramsey-Carnap method. Section 4 will clarify the role of mathematical terms in the distinction between observational and theoretical content. Then in Sect. 5, I will use this information to paint a general picture of how the application of mathematics in the sciences can be analysed using the Ramsey-Carnap method. Sects. 6-8 will incorporate a little chemistry by introducing infrared spectroscopy and applying the idea developed in the preceding sections. To finish, I will explain how my application of Ramseyfication relates to a possible solution for the Application Problem.

\section{The Application Problem of Mathematics}

Mark Colyvan's paper (2001) provides an extensive investigation of the Application Problem of Mathematics. Colyvan's main concern here is that the Application Problem is independent from any metaphysical stances one might have. I agree with him on this point. I will therefore refrain from getting involved in any deeper debate about structuralism; instead, I will stick to the grounds of Maddy's view-i.e. the

\footnotetext{
${ }^{4}$ Consequently, I will not be arguing the case for her view in this paper.

5 Other authors do this frequently. For instance, Patrick Suppes conflates formalisation and the application of mathematics while formalising theoretical vocabulary in Newtonian physics when he immediately includes explicit definitions of functions on real numbers. [See Suppes (1957, Sec.12.5).]
} 
warehouse which offers structures. However, as Colyvan explains, the Application Problem can be traced back to Wigner (1960), but was reformulated several times. Mark Steiner posed the problem as follows:

$[\mathrm{H}]$ ow does the mathematician - closer to the artist than the explorer - by turning away from nature, arrive at its most appropriate description? (Steiner 1995, p. 154)

Colyvan identifies several questions and subproblems that must be solved by a solution to the Application Problem. I will focus on the following two questions. ${ }^{6}$

1. How does the mathematician, by turning away from nature, arrive at its most appropriate description?

2. Why does mathematics lead to simpler theories and simpler calculations?

In a more recent paper, Daniel Waxman formulates the Application Problem as an explanatory challenge. [See Waxman (2021).] He identifies two components of this challenge, which are roughly aligned with Colyvan's questions.

(i) Pure mathematics is an a priori discipline that appeals essentially to aesthetic considerations. (Waxman 2021, p. 85)

(ii) Scientific practice draws substantially on mathematical concepts and structures. (Waxman 2021, p. 86)

The explanation provided by Waxman (2021) in response to this explanatory challenge is basically that mathematicians do not stray very far from a rather small set of structures which are naturally occurring in the sciences. The aesthetic aspects come into play when these structures are studied in an intelligible way. ${ }^{7}$ The applicability of any further development from these structures therefore comes as no surprise. Waxman tries to make his claim apparent by means of two examples: Galois Theory and Probabilistic Number Theory. I do not intend to argue in favour or against his claim here. ${ }^{8}$ Instead, I choose to tackle the Application Problem from a different angle, by trying to logically analyse the relations between mathematical structures and the data produced by measurement devices used in the natural sciences. I also try to make a case for this method through the successful application of Ramseyfication.

\footnotetext{
${ }^{6}$ Colyvan also argues in (2001, pp. 266-267) that any solution to the Application Problem must address the question of how mathematics plays a role in the predictions made by scientific theories. I disagree, because I see no reason why the case of a theory making predictions by mathematical means and the case of a theory making predictions without mathematics should be addressed separately. If an approach solves all the other aspects of the Application Problem, then the problems with predictions coincide with the single question of how any kind of theory, mathematical or otherwise, can lead to true predictions.

7 Waxman is rightly pointing out that the aesthetics follows a certain seriousness in mathematics, i.e. not everything is considered worth studying.

8 See Footnote 34 for a commentary on Waxman's thesis in the light of my analysis.
} 


\section{Ramseyfication: Technical Details}

At this point, I will depart from the standard presentation of Ramseyfication in the literature (especially in Definition 2); this departure will be justified in Sect. 4. To formalise a scientific theory, I will use a standard formal system for second-order logic with equality, comprising a set of formulas $\mathcal{F}(\alpha)$, which is formulated over a signature $\alpha$, a finite set of sentences $T$, i.e. the theory in a narrower sense, and a syntactically or semantically given consequence relation $\vdash .{ }^{9}$ I will denote such a formal system by $\langle\mathcal{F}(\alpha), T, \vdash\rangle{ }^{10}$ If there is a need to specify a semantics, one might choose between the three standard options for second-order logic, e.g. full, Henkin or frame semantics, taken from the Tarski tradition; for this text, however, it does not really matter, and further restrictions may be appropriate depending on the case in question. ${ }^{11}$ I separate the signature $\alpha$ into theoretical terms $\alpha_{T}$ and observational terms $\alpha_{O}$, such that $\alpha=\alpha_{O} \cup \alpha_{T}$ and $\alpha_{O} \cap \alpha_{T}=\emptyset$. [See Carnap (1956).] This bipartition of the signature induces a tripartition of the set of sentences from $\mathcal{F}(\alpha)$ into three categories: (1) theoretical sentences, i.e. sentences of $\mathcal{F}(\alpha)$ which lack occurrences of symbols from $\alpha_{O}$; (2) observational sentences, i.e. sentences of $\mathcal{F}(\alpha)$ which lack occurrences of symbols from $\alpha_{T}$; and (3) correspondence rules, i.e. sentences which include occurrences of both parts of $\alpha .{ }^{12}$

Definition $1 \mathrm{I}$ will refer to a formal system $\mathcal{T}=\left\langle\mathcal{F}(\alpha), T, \vdash_{\mathcal{T}}\right\rangle$ as a theory if $T$ is finite and comprises only theoretical sentences and correspondence rules.

I use $\mathcal{T} \vdash \varphi$ to express that $\varphi \in \mathcal{F}(\alpha)$ has a subset $\Phi \subset T$, such that $\langle\Phi, \varphi\rangle$ is in the consequence relation of the formal system $\mathcal{T}=\left\langle\mathcal{F}(\alpha), T, \vdash_{\mathcal{T}}\right\rangle$.

Definition 2 The observations $O_{\mathcal{T}}$ of a theory $\mathcal{T}$ are a finite set of observational sentences. $^{13}$

I refer to $\mathcal{O}_{\mathcal{T}}=\left\langle\mathcal{F}\left(\alpha_{O}\right), O_{\mathcal{T}}, \vdash_{\mathcal{O}}\right\rangle$ as a data-model of $\mathcal{T}$.

\footnotetext{
9 This is deliberately formulated without further restrictions. I see no reason for a metatheory at this stage. These notions are supposed to form a framework, covering as many theories as possible.

10 The choice of $\vdash$ is strongly related to the theory under consideration and one's aims. In Toppel (2020), I provided an extensive explanation of how to choose such a formalisation. I will leave this open here.

11 In Halvorson (2019) assumes that this does matter for Ramseyfication, but our discussion here goes beyond his scope.

12 Some readers might be confused by my decision to refer to these sentences as rules. But the reason why they are called by this name is because they formalise certain rules that scientists and laymen follow when connecting theoretical notions with measurement devices. Examples for such rules include looking at the thermometer to see the temperature, and the interpretation rules for infrared spectra mentioned in Sect. 7. Many philosophers see a close relationship between the rules outside of a formal system and the rules that define a calculus. Whether this relationship is indeed as close as propagated is up for debate. But it is not up for debate here. In this case, we only want to ensure that the formalisation of the rules followed by scientific practice are represented in a single sentence. This makes life easier when applying this apparatus.

13 Hence, the only requirement imposed by the theory on its observations is that they are reported in the same language.
} 
Note that observations can have any degree of logical complexity and that the consequence relations of $\mathcal{O}_{\mathcal{T}}$ and $\mathcal{T}$ do not have to coincide. In fact, I take these relations to be radically different. ${ }^{14}$ I will use $\mathcal{O}_{\mathcal{T}} \vdash \varphi$ analogously to $\mathcal{T} \vdash \varphi$.

To clarify how these formalisations are related to scientific practice, allow me to define the following notions, which are taken from (or inspired by) Lutz (2014a).

Definition 3 Assume $\mathcal{T}$ is a theory and $\mathcal{O}_{\mathcal{T}}$ its data model.

1. I say that $\mathcal{T}$ is empirically adequate with regard to $\mathcal{O}_{\mathcal{T}}$ if there is no $\varphi \in \mathcal{F}\left(\alpha_{O}\right)$ such that $\mathcal{T} \vdash \varphi$ and $\mathcal{O}_{\mathcal{T}} \vdash \neg \varphi$.

2. I say that $\mathcal{T}$ is empirically supported by $\varphi \in \mathcal{F}\left(\alpha_{O}\right)$ and $\mathcal{O}_{\mathcal{T}}$ if $\mathcal{T} \vdash \varphi$ and $\mathcal{O}_{\mathcal{T}} \vdash \varphi{ }^{15}$

If $\mathcal{T}$ is a theory that includes $T=\left\{\tau_{1}, \ldots, \tau_{n}\right\}$, then I take $\tau$ to be the conjunction $\tau_{1} \wedge \ldots \wedge \tau_{n}$. Note: since $T$ includes correspondence rules, observational and theoretical terms occur in $\tau$. I emphasise the occurrence of these terms in $\tau$ by $\tau(\vec{o}, \vec{t})$.

Definition 4 Assume $\tau(\vec{o}, \vec{t})$ is obtained from the theory $\mathcal{T}$.

The Ramsey sentence of $\mathcal{T}$ is

$$
\Re(\mathcal{T}) \equiv(\exists \vec{X}) \tau(\vec{o}, \vec{X}) .
$$

We also call this Ramsey sentence the Ramseyfication of the theory $\mathcal{T}$. The Carnap sentence of $\mathcal{T}$ is

$$
\mathfrak{S}(\mathcal{T}) \equiv \mathfrak{R}(\mathcal{T}) \rightarrow \tau
$$

Note that second order quantifiers occur in $\mathfrak{R}(\mathcal{T})$ when at least one theoretical term is a predicate or function symbol, which, of course, is usually the case. Also note that $\mathfrak{R}(\mathcal{T})$ is an observational sentence and that all consequences of $\mathfrak{S}(\mathcal{T})$ are logically valid. ${ }^{16}$

The following theorem is easy to prove:

Theorem 3.1 Assume that $\vdash_{T}$ is one of the standard second-order consequence relations. For any observational sentence, $\varphi$ holds that:

$$
\mathcal{T} \vdash_{T} \varphi \text { if and only if } \mathfrak{R}(\mathcal{T}) \vdash_{T} \varphi \text {. }
$$

Theorem 3.1, and the result that $\mathfrak{E}(\mathcal{T})$ is logically true, i.e. does not imply substantial truth, is the reason why some see $\mathfrak{R}(\mathcal{T})$ as the carrier of the empirically relevant

\footnotetext{
14 They can differ in their logical properties or the mathematical resources available to them. They might also model different causal relations.

15 Note that it is possible for a $\mathcal{T}$ to be both empirically inadequate and supported. For example, when research progresses and a new $\mathcal{O}_{\mathcal{T}}$ is formed, one which includes all the support given by a former data model but shows that the theory is empirically inadequate according to new findings.

16 The latter is easy to check. Proof can be found in Rozeboom (1962, pp.291-292).
} 
content of a theory which is structural in its nature. However, I am not going to argue the case for a version of structuralism in this paper. I identify the structural content of $\mathfrak{R}(\mathcal{T})$ with its ability to adopt a unique model-theoretical structure; I will not be drawing any ontological consequences here.

\section{An Extended Observational Language}

Carnap's observational-theoretical distinction reflects two contexts of verification (in the sciences) for a proposition, claim or sentence: either the verification proceeds through observations, e.g. through direct observations or measurements, or through an argument that draws on the theoretical background of the academic discipline. Noteworthy, mathematical vocabulary is frequently used in both contexts. Hence, Carnap includes these terms in observational sentences.

It is important to understand [...] that Ramsey's approach cannot be said to bring theories into the observation language if "observation language" means (as is often the case) a language containing only observational terms and the terms of elementary logic and mathematics. (Carnap 1966, p. 252)

This quotes confirms that Carnap includes elementary notions of mathematics in his weakest version of an observational language. This is a version he is going to extend even further through the need for second order quantifiers to match the syntax of the Ramsey sentence. Carnap proceeds by introducing the notion of an extended observational language. He extends the realm of possible referents for observational sentences to match the needs of the second order quantifiers. The fact that there are, therefore, no syntactical restrictions on the length or order of quantification in observational sentences was pointed out by Lutz (2014a, p. 1173) and is something I implicitly used myself in Sect. 3; hence, this is the promised justification for Definition 2. However, I consider this uncontroversial. What I wish to pinpoint is the interrelation between the occurrence of elementary mathematical notions in the observational sentences alongside second order quantifiers. The combined occurrence of these elements is the reason why I called an $\mathcal{O}_{\mathcal{T}}$ a data model, because it allows for the introduction of continuous mathematics and statistical methods into $\mathcal{O}_{\mathcal{T}}$. I like to think of the corresponding $\vdash_{\mathcal{O}}$ as extending logic to a mathematical consequence relation, which might be chosen as a constructive system, e.g. intuitionistic mathematics or characterisations of computability classes or such. I will explore where this thought might lead in the following section.

\section{Applying Mathematics in the Sciences}

In order to show how the application of mathematics can be understood with the apparatus described in the previous sections, let us start with a simple example that closely relates to Carnap's discussion in (1966, p. 225): 


\section{(*) This rock has a temperature of twenty centigrade.}

The phrase "twenty centigrade" is (or rather, should be formalised as) an observational term, since it can be easily measured using a manual device such as a thermometer. Also, the number term 20 is elementary enough to count as observational in the sense of a verification context as distinguished at the beginning of Sect. 4. ${ }^{17}$ The noun-phrase "this rock" can be formalised as an observational term without causing controversy, whereas "temperature" is a theoretical term. ${ }^{18}$ Hence, (*) should be formalised as a correspondence rule. ${ }^{19}$ It is tempting to formalise this statement as

$$
T(r)=20 .
$$

However, this would be wrong because no temperature is identical to a number. According to modern physics, temperature is a configuration of the movements of the particles which are aggregating to a material body. To view temperature as a number is to relate these configurations to those of another body by a scale. ${ }^{20} \mathrm{I}$ consider it most appealing to specify the scale by using an operator, as in

$$
{ }^{\circ} C[T(r)=20] \text {. }
$$

Of course, the need for this becomes more apparent when more than one measurement device with different units is in use. I did not include this in Sect. 3, because I have no characterisation of their logic and one of the aims of this paper is precisely to show that the logic of these operators does not have to be spelled out. So we keep working with $T(r)=20$ as the formalisation. ${ }^{21}$ Note that this problem only occurs in the case of correspondence rules, because the unit only denotes a logical function when combined with a theoretical term like temperature or mass. Furthermore, methodologically speaking, ignoring this aspect of the sentence is not a problemas will be made clear later. It is just a syntactical change (which was skipped in Sect. 3) from modal to pure first-order that would have appeared before the step discussed here. By this I am referring to the step from first-order to the Ramsey sentence, which is a second-order sentence.

\footnotetext{
17 Of course, one might wonder how big a natural number can be or how the denominator of a fraction can be such that a number term still counts as a elementary notion of mathematics. Discussions such as those in Suppe (1972) are to be avoided. As long a measurement device is fixed, the situation is rather clear: readability on the scale or the limits of the display will clarify on the limits of whether a number term counts as observational

18 Carnap also explicitly states this in (1956, p. 48). It is also clear from the requirement that $\alpha_{O} \cap \alpha_{T}=\emptyset$ and the fact that "temperature" frequently appears in theoretical contexts.

19 Carnap also implies this in (1956, p. 48). Readers who are well-acquainted with Carnap should not be confused by the fact that Carnap also calls this sentence an empirical law, for this apparent conflict of notions can be resolved by distinguishing between a pre-formalised sentence and a formalised sentence. [See Toppel (2020, pp. 34-35.)].

${ }^{20}$ For instance, when a thermometer was calibrated by being previously applied to a body with known temperature.

21 Here the opposition to those using mathematical entities as intended referents becomes effective, as it was promised in the introduction.
} 
Since $T(r)=20$ is a correspondence rule, after Ramseyfication it is identified with

$$
\exists f[f(r)=20] .
$$

This is an observational sentence. Whatever the semantic value of $T$ was before, after Ramseyfication we are free to take whatever we want for $f$ 's value. We are only restricted by the condition that $r \mapsto 20$. Hence, depending on how objects are formalised into language, if these objects are represented by coordinates then the choice for such a function can be viewed as a mathematical regression (or any other kind of statistical modelling).

Rephrasing this in the terminology of Definition 3, we can say the following:

A case of empirical support for a theory $\mathcal{T}$ can be made when two things happen:

1. $\mathfrak{R}(\mathcal{T}) \vdash \exists f \varphi(f)$

2. $\mathcal{O}_{\mathcal{T}} \vdash \exists f \varphi(f)$

Theorem 3.1 assures that (1.) does not go beyond the scope of Definition 3. Additionally, the use of different consequence relations in (1.) and (2.) might yield further informative results for theories under investigations. This is because the consequence relation in (1.) is some suitable fragment of classical second-order logic provided by a system of deduction or customary semantics, whereas the consequence relation in (2.) is not purely logical. That is to say, it may incorporate basic causal relations and statistical methods, and it should proceed constructively. ${ }^{22}$

(1.) tells us that in any possible reinterpretation of $\mathcal{T}$, one can find a function that satisfies the conditions expressed by $\varphi$, which only includes occurrences of symbols from $\alpha_{O}$.

In cases where $\alpha_{O}$ comprises only denotations for numbers (and maybe easy functions on these numbers), this allows for a change of reference for $\alpha_{T}$, from concrete entities (like atoms) to entirely abstract entities (like numbers). ${ }^{23} \mathfrak{R}(\mathcal{T})$ allows for a change of reference to a richer structure previously provided by mathematics. ${ }^{24}$ The richer structure actually allows a function such as $f-$ e.g. the solution to an eigenvalue equation in quantum physics-to be found. But if the function is found and its existence can be proven by $\mathfrak{R}(\mathcal{T})$, then it can be justified by $\mathcal{T}$ alone by means of Theorem 3.1. ${ }^{25}$ This also avoids issues with the different logical requirements for

\footnotetext{
${ }^{22}$ The requirement to proceed constructively does not reflect a philosophical stance; it is just a matter of fact that data analysis in the sciences is carried out by effective algorithms, e.g. when a working scientist analyses data with a computer programme.

${ }^{23}$ I agree with Halvorson when he says that:

To the contrary, logical semantics is... wait for it... just more mathematics.

(Halvorson 2019, p. 15)

But of course, this is on the metalevel. It still makes sense to say that "Hans" denotes a particular professor of the formal philosophy of science and that "1" denotes the number one.

${ }^{24}$ See Maddy's (2008) for the underlying picture of mathematics adopted here.

${ }^{25}$ Although this requirement might sound strong, notice that it is satisfied by the example of infrared spectroscopy.
} 
units that may appear in the correspondence rules, because changing the reference to an abstract object eliminates the dependency of a predefined scale. The theory $\mathcal{T}$ is then empirically supported when the data allows a suitable (similar enough) function to be found, i.e. when $\mathcal{O}_{\mathcal{T}}$ finds a function that satisfies the same conditions (those expressed by $\varphi$ ).

This leads us to an answer for Steiner's question:

How does the mathematician, by turning away from nature, arrive at its most appropriate description?

The answer is: she does not. As Maddy described in (2008), the mathematician only provides mathematical structures that can be applied in the sciences. But the theory provides a separate structure, and mathematics is only tied to this latter structure through the fixture that is provided by the mathematical terms in the observational language. However, this tying is obviously highly interpretative, i.e. the mathematical structure can only act as a mathematical model when it simultaneously satisfies two conditions: (1) it must fit the data model, and (2) it must embody the structure provided by the theory. Hence, there is no epistemological gap to be bridged.

I would like to close this section by claiming that the most interesting cases include observational languages which are entirely constructed on the basis of mathematical notions. I claim this because (1) the language of measurements is entirely mathematical in scientific contexts, and (2) the application of mathematics-as described above-seems most appropriate when this language is used. This will become clearer when such a language of measurements is studied in relation to its measurement technique in the following sections.

\section{Infrared Spectroscopy}

It seems like I did two bad things. First, I gave a "solution" to the Application Problem of Mathematics, which is just basic model theory. Second, I identified a problem with the logic of unities, which I then solved immediately by using again basic model theory. One might therefore say that I artificially constructed a problem.

In order to show that something of substance is revealed when Ramseyfication is considered (or used) in this way, I will use the method on infrared spectroscopy. However, the range of the quantifier in the existence claim as described in Sect. 5 does not include functions but only single values, when the data is given as an infrared spectrum.

Infrared spectroscopy is a standard measurement technique that has many applications in organic chemistry. It is mainly used in the qualitative analysis of chemicals, i.e. to identify the chemical or the components of a mixture. The basic principle of this technique is that heat causes vibrations in the atoms of a molecule. Also, sub-molecular structures can only remain in discrete quantum stages that correspond to the energy levels they are able to absorb. Moreover, since energy corresponds to the wavelength of radiation and heat corresponds to infrared radiation, wavelengths correspond to sub-molecular structures. Now the idea behind infrared spectroscopy is to measure the wavelengths that are not absorbed in order to draw conclusions 
about the wavelengths that have been absorbed. This data allows the chemist to ascertain certain properties of the sub-molecular structure. Since the bond must be stable in order to move, this method works best with atomic bonds, e.g. bonds between two carbon atoms $(C-C)$, a carbon and a hydrogen atom $(C-H)$, a carbon and an oxygen atom $(\mathrm{C}-\mathrm{O})$, and an oxygen and a hydrogen atom $(\mathrm{O}-\mathrm{H})$. This is why it is widely applicable in organic chemistry. The type of movement that infrared radiation induces in a molecule is the vibration of the atoms along their bonds. There are six types of vibrations to be distinguished in a molecule; however, these need not concern us here. The data produced by the measurement devices are points that pair a wavelength $(\lambda)$ in centimetres $(\mathrm{cm})$ with the intensity $(I)$ of the corresponding radiation. However, it proves rather useful to consider the matter of

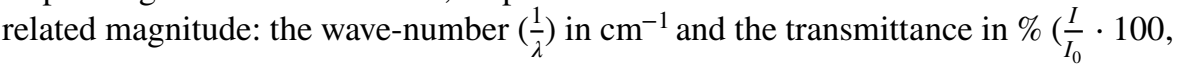
with $I_{0}$ as the original intensity). When the data is displayed in a suitable diagram and the lines are connected, a curve is obtained. Below is the curve for ordinary alcohol (ethanol), which has the following structure:<smiles>CCO</smiles>

The (downward) peaks corresponds to an atomic bond. For instance the huge peak at the beginning, i.e. between 3500 and $3000 \mathrm{~cm}^{-1}$, is a sign for $O-H$. The sharper peak close to $3000 \mathrm{~cm}^{-1}$ is a sign for $H-C-H$. 


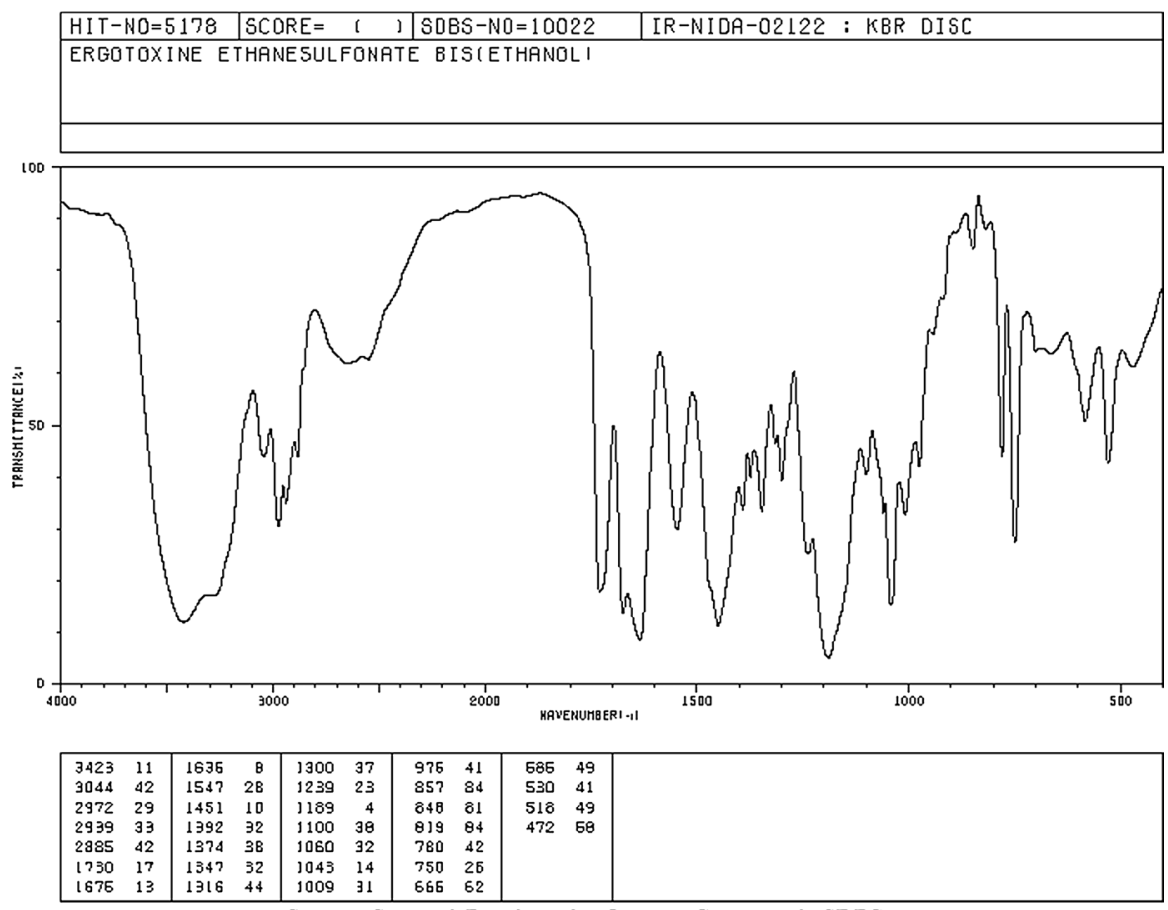

Source: Spectral Database for Organic Compounds SDBS,

hetps://sdbs.db.aise.go.jp/sdbs/cgi-bin/landingpage?sdbsno $=10022$

I consider it important and essential to the theory behind this measurement method that a chemist does not have to know any quantum physics in order to use this measurement technique competently, i.e. to interpret a spectrum. Also, there is no need for mathematics as yet. All there is, is a taxonomy of chemical bonds and their possible vibrations, which correspond to wave numbers.

Let me pre-empt two possible counter-arguments against my decision to use this example. I can think of two such arguments, the most common probably being that I am ignoring the fact that the theory of infrared spectroscopy is deducible from quantum mechanics. However, this is wrong. The taxonomy of the vibrations must be used when formulating and solving the eigenvalue equations. The logic of the unmathematical theory therefore precedes that of the mathematical theory. [See Bright Wilson et al. (1980).] Others might argue that the theory I have presented so far is merely a degenerative form of a more advanced theory which is highly mathematical and basically equates to applied quantum mechanics. I agree, but this does not make the theory, as I present it, any less of a theory in its own right. Indeed, it is taught, used and applied in this form in an academic context.

Moreover, coming from the Ramsey-Carnap tradition, it is not that unnatural to look into spectroscopy. As one of the closest connections between the unobservable micro-structure of the world and the data scientists gather about it, spectroscopy is the natural place to look for correspondence rules. 


\section{Applying Ramseyfication to Infrared Spectroscopy}

Of course, formalising the theory of infrared spectroscopy is not possible in the narrower scope of this paper; moreover, it is also extremely boring. Instead, I rely on the reader's imagination and the suggestion that a molecule can be easily represented as a finite connected graph whose edges have finitely many properties. It is easy to see that all the taxonomies given in a standard textbook on IR spectroscopy can be spelled out in a second-order system. ${ }^{26}$ The theoretical sentences should comprise the following information:

1. They should state that the smallest components of a molecule are atoms and that anything that is composed of molecules is also a molecule.

2. They should state which bonds can appear between certain atoms and which types of vibrations these bonds can perform, and state certain connections between these vibrations.

3. They might also include more advanced parts of the theory, i.e. how vibrations change when intermolecular changes occur, including a theory of hydrogen bonds and so on.

In order for this to count as a theory in the sense of Definition 1, we also need correspondence rules. These should express the standard interpretation rules for spectra as given in the textbooks (like Hesse et al. 2012), e.g. "A $(H-C-H)$ peaks at between 3050 and $2850 \mathrm{~cm}^{-1}$, 27

There are also certain aspects that help us with the task of formalising a data model in the sense of Definition 2; note that 4001 is a prime number and the standard measurement devices only produce data in the natural numbers. Hence we need only number terms for natural numbers up to 4000 and we get a field-and therefore all of basic arithmetic - for free. ${ }^{28}$ On the other hand, there is no need for number terms at all in the theoretical vocabulary, because the formalisation of molecules as finite graphs means that alternating existence quantifiers can perform any counting required, e.g. for chemical formulas like $C_{x} H_{y} O_{z}$.

Coming back to the observational language, a spectrum can be formalised as a mere list of peaks, e.g. as a finite list of pairs of numbers, the first of which denotes the wave number while the second indicates the magnitude of the peak. After all, the graph above purely benefits human cognition; the same information is provided in the list below the graph.

At this point, the example already shows the extent to which number terms can be taken as observational: there is no problem of epistemological accessibility in recognising that the first peak is at $3423 \mathrm{~cm}^{-1}$ in the spectrum of ethanol. In other words, it is epistemologically unproblematic to verify that

\footnotetext{
${ }^{26}$ For any sceptics I offered some suggestions in this regard in Toppel (2020).

27 See the tabular in Hesse (2012, p. 51).

28 Because the ring of integers modulo 4001 then becomes a field.
} 


$$
\exists x[S(x) \wedge \operatorname{Ethanol}(x) \wedge p(1, x)=3423],
$$

where $S(x) \wedge \operatorname{Ethanol}(x)$ says "is a spectrum of ethanol" and $p$ is a function denoting the wavenumber for the first, second peak (and so on) in a spectrum, is true; therefore, to call it observational is justifiable.

So, I provided a good example of a formalisation of a scientific theory, with the particularity that mathematics only occurs in observational sentences. It also makes a clear and uncontroversial distinction between epistemologically problematic and epistemologically unproblematic sentences when it comes to verification or accessibility. Note that we only need three types of entities here:

1. Natural numbers between 0 and 4000 .

2. Spectra, i.e. a finite list of pairs of numbers. ${ }^{29}$

3. Atoms and molecules.

The first two entities are presupposed by the observational sentences, whereas the third is only presupposed by the theoretical sentences. Chemicals are not required as an ontological basis in addition to their spectra and their molecular structure. ${ }^{30}$

It is usually considered a malfunction of formal systems that they cannot specify their models up to isomorphism, whereas this is the main benefit of formalisation for our purposes here. Since the theory can have models of any cardinality, we can construct a structure based on the real numbers; one that satisfies the theory but adds more structure. This is what it means to use mathematics in the case of this theory. The structure added by the mathematics can then be used for theoretical purposes.

\section{Applying Mathematics to the Theory}

We have a choice here. We can either opt for the mathematics that classical physics developed by using a quantified version of the harmonic oscillator (like in Hesse et al. 2012), or we can incorporate the entire mathematical apparatus that contemporary quantum physics has to offer (as in Bright Wilson et al. 1980). Based on my view on the application of mathematics, it should suffice for us to work with the classical version as described in Hesse et al. (2012).

We start by identifying atoms with the (numerical) value of their atomic mass. Hence our ontology changes from $H, C, N, O$ and $S$ to $1,12,14,16$ and $32 .^{31}$ Identifying atoms with the value their atomic mass does not disregard important links

\footnotetext{
29 Their type-token distinction is no more epistemologically or metaphysically problematic than that of any entity that can be printed.

${ }^{30}$ This does not mean that I am concluding that the material basis of the measurement technique is reducible. I want to recall that we just picked out one identifiable portion from a collection of scientific theories in organic chemistry. This theory is not intended to stand on its own.

31 I have used rounded values for the sake of simplicity. Note that these are not number terms; they are numbers that serve as the reference for variables which are quantified by an existence quantifier in the Ramsey sentence.
} 
between the notion of mass and mathematics. Indeed, infrared spectroscopy does not explicitly talk about mass in addition to the quality of an atom or its vibrations, so this does not appear to be relevant in terms of its mathematical modelling when it comes to this particular context of verification. If I failed to include this aspect, then it is not because I took a shortcut, but because it can be eliminated in this particular case. After reinterpretation, both "mass of 12 " and "carbon atom" become "is 12 ".

Adequate mathematical objects must be chosen as references for the other variables in Ramseyfication depending, in particular, on how molecules have been formalised. For explanatory purposes, I use triples of numbers $\left(k, m_{1}, m_{2}\right)$ for bonds, where $k$ encodes the quality of the bond and the $m_{i}$ s are either $1,12,14,16$ or 32 . Of course, additional axioms must be added to the Ramsey sentence so that both the references of the variables are specified and suitable operations can be performed. The details should not be hard to estimate. This allows us to add new structure by adding one further axiom to the Ramsey sentence of infrared spectroscopy:

if $\left(k, m_{1}, m_{2}\right)$ is a bond, then

$$
(* *) \quad k=\frac{m_{1} \cdot m_{2}}{m_{1}+m_{2}} \cdot\left(\frac{\tilde{v}}{1302}\right) .
$$

This formula is derived from Hooke's law, where a bond is viewed as a harmonic oscillator. ${ }^{32}$ Note that the correspondence rules took the form

"A $(H-C-H)$ peaks at between 3050 and $2850 \mathrm{~cm}^{-1}$ ",

This is how they look after Ramseyfication and mathematisation:

"For any $x$, such that $(\mathrm{x}, 1,12)$ is a bond, $(* *)$ gives a value $\tilde{v}(x, 1,12)$ such that $3050 \leq \tilde{v}(x, 1,12) \leq 2850$."

The correspondence rules are then either consistent or not. If they are consistent, then the mathematical model is adequate. Of course, whether the mathematical model is adequate depends on how narrow ranges such as "3050 $\leq \tilde{v}(x, 1,12) \leq 2850$ " are taken to be.

To link this to the discussion in Sect. 5 regarding the formula $\exists f \varphi(f)$, the required existence claim here only concerns a value provided by the function $\tilde{v}(x, 1,12)$ and not the function itself. It has the form

$$
\exists y \varphi(3050 \leq y \leq 2850) .
$$

This formula can be proven by the theory because we assume that there are certain bonds in a molecule, and it can be proven by the data model because a value can be read off a spectrum by the function $p$ from Sect. 6 .

$\overline{32}$ I explained this in detail in Toppel (2020). 


\section{Conclusions}

In this paper, I upgraded the presentation of Ramseyfication to make it more applicable to modern science, and I applied the approach to the method and theory of infrared spectroscopy. I sketched a formalisation of the theory of infrared spectroscopy $\mathcal{T}_{I R}$ as a kind of finite graph theory with extra information about edges. I built a data model in the sense of Definition $2 \mathcal{O}_{\mathcal{T}_{I R}}$, which is basically a theory about a finite set of finite lists. I then extended $\mathfrak{R}\left(\mathcal{T}_{I R}\right)$ with the addition of $(* *)$ and basic arithmetic.

Since the observation language of $\mathcal{T}_{I R}$ was formed entirely of mathematical terms, the canonical model for $\mathfrak{R}\left(\mathcal{T}_{I R}\right)+(* *)+$ (some basic math) is a structure that comprises only numbers and other abstract objects.

The above shows that Ramseyfication, has something valuable to say about an unsolved philosophical problem: in this case, the Application Problem of Mathematics mentioned in Sect. 2. Mathematicians provide mathematical structures. Applying mathematics in the sciences means tying these structures to the structural content given by the Ramsey sentences of a theory in question. This tying is interpretative in the sense that the mathematical terms in the observational language provide a fixture in which these structures have to fit. ${ }^{33}$ This interpretive aspect of the application is the reason why no epistemological gap arises between abstract objects and concrete objects, since all structures of relevance to concrete objects were already present in the theoretical part of the theory. ${ }^{34}$ As mentioned in Sect. 4, the distinction between a theoretical language and an observational language reflects the distinction between the contexts of justification for claims, propositions or sentences. Since mathematical terms cannot be separated from their observational context, such terms must remain in the observational language. However, the example of infrared spectroscopy shows that the situation is different in the case of higher mathematics and the theoretical content of a theory. More elementary (or constructive) concepts are integral to the observational part of the language, which allows higher mathematics to be applied to the theoretical content and removed as required, provided two factors are taken into account. Firstly, the mathematics must satisfy the structural needs of the theoretical content (as given by the Ramsey sentence) and, secondly, its structure must match the outline demanded by the elementary mathematics in the observational part.

I see this as a convergence between a more developed version of Ramseyfication and the position expressed by P. Maddy's claim that higher mathematics and the sciences are not as strongly intertwined as Quine and Putnam believed or as argued by the indispensability argument for mathematical realism. [See Maddy (2007, IV.2).] When it comes to the sentences of mathematics and theoretical physics,

\footnotetext{
${ }^{33}$ This might be seen as a criticism of structuralism, because not only is finding a structure highly interpretive, but the structural content (whatever this is) also changes in relation to the chosen units, i.e. one might view the theory of organic molecules from the perspective of mass spectroscopy instead of infrared spectroscopy.

${ }^{34}$ Note, for instance, that the basic taxonomies of vibrations, which presuppose a structure, must be given in order to formulate the eigenvalue equation in the case of quantum physics. (See Bright Wilson et al. 1980.) Whether this supports or disclaims Waxman's thesis as presented in Waxman (2021) cannot be decided without further analysis of the structures presupposed by the theories. But the problem identified in Footnote 33 seems to raise a challenge for Waxman.
} 
mathematical notions might be superficially connected to theoretical terms by the scientist's linguistic practice, but that does not make them logically inseparable.

Funding Open access funding provided by University of Vienna.

Open Access This article is licensed under a Creative Commons Attribution 4.0 International License, which permits use, sharing, adaptation, distribution and reproduction in any medium or format, as long as you give appropriate credit to the original author(s) and the source, provide a link to the Creative Commons licence, and indicate if changes were made. The images or other third party material in this article are included in the article's Creative Commons licence, unless indicated otherwise in a credit line to the material. If material is not included in the article's Creative Commons licence and your intended use is not permitted by statutory regulation or exceeds the permitted use, you will need to obtain permission directly from the copyright holder. To view a copy of this licence, visit http://creativecommons.org/licen ses/by/4.0/.

\section{References}

Bright Wilson, E., Decius, J. C., \& Cross, P. C. (1980). Molecular vibrations. The theory of infrared and Raman vibrational spectra. New York: Dover Publications.

Carnap, R. (1956). The methodological character of theoretical concepts. Minnesota Studies in the Philosophy of Science, 1, 38-76.

Carnap, R. (1966). An introduction to the philosophy of science. New York: Basic Books.

Colyvan, M. (2001). The miracle of applied mathematics. Synthese, 127, 265-277.

Halvorson, H. (2019). The logic in philosophy of science. Cambridge: Cambridge University Press.

Hesse, M., Meier, H., \& Zeeh, B. (2012). Spektroskopische Methoden in der organischen Chemie (8th ed.). Stuttgart: Georg Thieme Verlag.

Lutz, S. (2014). Empirical adequacy in the received view. Philosophy of Science, 81, 1171-1183.

Lutz, S. (2014). What's right with a syntactic approach to theories and models? Erkenntnis, 79, $1475-1492$.

Lutz, S. (2017). Carnap on empirical significance. Synthese, 194, 217-252.

Maddy, P. (2007). Second philosophy. Oxford: Oxford University Press.

Maddy, P. (2008). How applied mathematics became pure. The Review of Symbolic Logic, 1(1), 16-41.

Psillos, S. (1999). Scientific realism: How science tracks truth. London: Routledge.

Rozeboom, W. W. (1962). The factual content of theoretical concepts. In H. Feigl, M. Scriven, \& G. Maxwell (Eds.), Scientific explanation, space, and time (pp. 273-357). Minneapolis: University of Minnesota Press.

Steiner, M. (1995). The applicabilities of mathematics. Philosophia Mathematica, 3, 129-156.

Suppe, F. (1972). What's wrong with the received view on the structure of scientific theories? Philosophy of Science, 39(1), 1-19.

Suppes, P. (1957). Introduction to logic. Cincinnati: Litton Educational Publishing.

Suppes, P. (2002). Representation and invariance of scientific structures. Stanford: CSLI Publication.

Toppel, M. (2020). A solution to the application problem of mathematics by Ramseyfication. MA-Thesis, University of Vienna.

Uebel, T. (2011). Carnap's ramseyfication defended. European Journal of Philosophy of Science, 1, 71-87.

Waxman, D. (2021). Is mathematical unreasonably effective? Australian Journal of Philosophy, 99, 83-99.

Wigner, E. P. (1960). The unreasonable effectiveness of mathematics in the natural sciences. Communications on Pure and Applied Mathematics, 13, 1-14.

Publisher's Note Springer Nature remains neutral with regard to jurisdictional claims in published maps and institutional affiliations. 